\title{
ESTILOS EXPLICATIVOS Y HABILIDADES PARA LA GESTIÓN DE NEGOCIACIÓN DE CONFLICTOS EN ADOLESCENTES ESPECTADORES DE VIOLENCIA ENTRE PARES (BULLYING)
}

\author{
Explanatory styles and management skills for negotiation of conflicts in \\ adolescent spectator violence among peers
}

\author{
Alberto Quintana P. ${ }^{1}$, William Montgomery U., Carmela Malaver S., Gabriela Ruiz S. \\ Universidad Nacional Mayor de San Marcos, Lima, Perú
}

(ReCibido el 22/03/2011 - AcEPtAdo el 09/06/2011)

\begin{abstract}
RESUMEN
Se estudiaron las relaciones entre los estilos explicativos y las habilidades de gestión para la negociación de conflictos en adolescentes espectadores de bullying, con el objetivo general de precisarlos y, en adelante, proponer alternativas de prevención de este problema. Para la obtención de datos se utilizó: el Cuestionario de Estilos Atributivos, el Test de Habilidades para la Gestión en la Negociación de Conflictos y la Escala para Identificar el Tipo de Espectador de Violencia Entre Pares. Se contrastó las hipótesis mediante un diseño de tipo descriptivo-comparativo, con un muestreo circunstancial de la población a estudiar. Para cuantificar los resultados se usó el análisis de varianza encontrando diferencias significativas entre los tipos de espectadores. También se uso el coeficiente de Pearson para establecer las correlaciones entre las variables estudiadas, encontrándose que existe una correlación directa y significativa entre el espectador prosocial y todas las habilidades para la gestión de conflictos interpersonales, mientras que, por el contrario, los espectadores amoral e indiferente-culpabilizado no guardan correlación con estas habilidades, a excepción de la de compromiso con la cual su correlación es inversa y significativa. De otro lado, el espectador prosocial correlaciona positiva y significativamente, con atribuir a su propio esfuerzo y habilidad sus éxitos, y los espectadores amoral e indiferente-culpabilizado, por el contrario, correlacionan significativamente con atribuir a causas ajenas a sí mismos sus éxitos y fracasos, así como a atribuirse a sí mismos sus fracasos.
\end{abstract}

Palabras clave: Adolescentes, espectadores de bullying, gestión de conflictos, estilos explicativos.

\begin{abstract}
It was Studied the relationships between explanatory styles and management skills for negotiating conflicts in bullying young viewers, in order to clarify and, in future, propose alternatives to prevent this problema, on the understanding that, as previous studies have concluded, explanatory style that everyone argues about their responsibility in the development
\end{abstract}

1 Docente principal de la Facultad de Psicología de la UNMSM. E-mail: albertolqp@ yahoo.es 
of interpersonal conflict is linked to their own ability to handle them. To obtain the data was used: the attributional styles questionnaire, the Test Management Skills in Negotiation of Conflict, Scale to identify the type of viewer Peer Violence. Hypothesis was tested using a descriptive design-comparative, with a sampling circumstantial the study population. To quantify the results using analysis of variance found statistically significant differences between the types of audiences in 4 of 5 attributional styles and 3 of the 5 skills to manage interpersonal conflicto. This also used the Pearson correlation coefficient to establish correlations between these variables. found as postulated hypotheses, there is a direct and significant correlation between the prosocial viewer and all skills to manage interpersonal conflicts, whereas, by contrast, blamed amoral viewer and indifferent spectator do not correlate with these skills, except for the commitment with which the correlation is inverse and significant. On the other hand, as postulated, the prosocial spectator correlates positively and significantly to attribute their own successes to his effort and skill, and amoral and indifferent blamed spectators, correlate significantly to attribute causes of successes and failures other than themselves, and to attribute their failures to themselves.

Keywords: Adolescents, spectators of bullying, conflict management, explanatory style.

\section{INTRODUCCIÓN}

La violencia entre pares, o bullying, entendida como una manifestación de agresión infantil y juvenil en el marco del acoso escolar, actualmente se encuentra en la mira del profesional especializado, con el fin de prevenir tempranamente su aparición o impedir su mantenimiento (Quintana, Montgomery y Malaver, 2009; García, 2008).

Dado que en nuestras anteriores investigaciones hemos enfatizado la detección tanto del espectador de bullying cuya indiferencia, tolerancia o atención refuerza o no castiga tal forma de violencia, como de aquellos que por el contrario apoyan a la víctima y confrontan al bullying, ahora abordamos el problema de sus repertorios cognoscitivos en relación con su conducta instrumental, para precisarlos objetivamente y a partir de allí proponer alternativas comportamentales eficientes, en términos de fundamentar la promoción entre los espectadores de violencia entre pares de un repertorio de estilos explicativos coherentes con una práctica eficaz de solución de conflictos interpersonales.

A partir de estas consideraciones nos planteamos la siguiente interrogante: ¿Cuál es la relación entre los estilos explicativos y las habilidades de gestión para la negociación de conflictos en adolescentes espectadores de bullying?

\section{Estilos explicativos}

Resulta indiscutible que hay una relación determinante entre las concepciones que un individuo tiene del mundo y el modo como se comporta, y que ésta relación tiene que ver especialmente con los repertorios conductuales del lenguaje y la motivación. En este sentido, una persona aprende formas muy complejas de conducta verbal empapadas de valor emocional, ligadas a clases de acciones y habilidades instrumentales (Staats, 1997). Por ello es que los procesos interpretativos manejados por el sujeto sobre las causas de ciertos acontecimientos pueden modular su conducta. A esta facultad de autorregulación se 
le ha llamado desde diferentes perspectivas constructos de "locus de control", "autoeficacia percibida" o "estilo atribucional" (Visdómine-Lozano y Luciano, 2006), involucrando en todos los casos las hipótesis del individuo sobre la causalidad de los eventos con los que interactúa en función o bien del esfuerzo responsable de su conducta personal o bien de situaciones externas a sí mismo.

\section{Habilidades para la gestión de negociación de conflictos}

Dado el carácter social de las interacciones humanas, dentro del espectro de comportamientos instrumentales relevantes para el desempeño está la capacidad de negociación de conflictos con nuestros congéneres, en la cual juegan funciones muy importantes como el control emocional y otras habilidades (Malaret, 2001). Es de esperar, que el estilo explicativo que cada uno tenga acerca de su responsabilidad sobre la causalidad de los acontecimientos esté relacionado con su propia capacidad de manejo de conflictos interpersonales, tal como se ha visto en multitud de estudios, entre los cuales se pueden mencionar los recientes sobre la ansiedad y la agresión (Linares, 2007), y las estrategias de afrontamiento (San Juan y Magallares, 2007)

\section{Espectadores de violencia entre pares (bullying)}

Los estudiosos de la violencia entre pares o bullying (Monjas y Avilés, 2006: 31) la definición como la actividad de tipo agresivo que algunos escolares -apoyados por la inhibición o tolerancia, e inclusive el aliento, del grupo- aplican repetida y deliberadamente sobre otros alumnos de forma sistemática, utilizando modalidades físicas, verbales y sociales. La dinámica de la violencia entre pares es la relación establecida entre los tres actores que participan en la situación de maltrato: agresor, víctima y espectador, caracterizándose por una asimetría en la cual los agresores intentan someter a la víctima y convertir al espectador en un cómplice activo o silencioso que tolera esta situación (Avilés, 2006).

El agresor suele ser un individuo con fortaleza física mayor, con falta de empatía afectiva, sin sentimientos de culpa, muestra un estilo atribucional externo, no es socialmente competente, trasgresor, con estilo agresivo activo al interrelacionarse intimidando directa o indirectamente. Por el contrario, la víctima es de menor fortaleza física, puede incluso presentar discapacidades, diferencias por pertenecer a minorías étnicas o sociales, con baja autoestima, timidez, inseguridad e inhabilidad social, careciendo a menudo de una red de apoyo, con características sumisas, pasivas, dependientes e inasertivas, con fracaso escolar, y en algunos casos pudiendo mostrar conductas provocadoras o desafiantes.

Y distinguen entre los espectadores, tres tipos diferenciados:

a. Antisociales: no actúan directamente, pero refuerzan de alguna manera la conducta del matón, por ejemplo, sonriendo o con gestos cómplices. Están complacidos y a veces justifican el maltrato... Algunos pueden tener dudas 
y sentirse mal, pero se doblegan a las presiones del grupo o del matón, ya que tienen miedo ellos mismos de ser objeto de burlas, intimidación o verse fuera del grupo...

b. Espectadores propiamente dichos: observan y no hacen nada; permanecen "neutrales" y pueden sentirse indiferentes con la situación, no dándole importancia en absoluto, con lo que toleran y se acostumbran a estas injustas situaciones. A veces se muestran nerviosos e incómodos/as, pero no hacen nada...

c. Prosociales: ayudan a la víctima y hasta pueden llegar a recriminar al agresor, verbal o gestualmente: "macho, te has pasao", "tía, ya te vale“. Se sienten afectados y a veces asustados y suelen pedir ayuda y/o comunicárselo a los adultos.

Puesto que nuestro grupo de investigación se viene especializando en el estudio de los casos de conflicto interpersonal en el entorno de bullying adolescente, y especialmente enfocado en la condición del espectador de esta forma de violencia —en sus diferentes tipos - como uno de los factores que posibilita el acoso escolar en términos de refuerzo social (Avilés, 2006; Quintana, Montgomery y Malaver, 2009), creemos que la relación postulada entre estilos explicativos y capacidad de negociación tiene implicancias prácticas. En efecto, si se determina con precisión qué estilos explicativos se hallan presentes en la tolerancia o aliento del espectador hacia el acoso, y cuáles de ellos implican pensamientos y sentimientos sobre la propia capacidad de evitarlos o intervenir de alguna manera, podremos articular procedimientos de cambio centrados en la promoción o auspicio de conductas instrumentales que contrarresten la aparición de estas dificultades, es decir, promocionar adolescentes con estilos explicativos resilientes alternativos y coherentes con una práctica interpersonal plena de capacidad de disfrute, apoyo comunitario percibido y habilidades de negociación de conflictos.

Actualmente se dispone de instrumentos que sirven para evaluar los constructos mencionados. Entre ellos, se seleccionarán tres que cuentan con la ventaja de haberse elaborado o adaptado a nuestro medio: el Cuestionario de Estilos Atributivos (CEA) (Alonso, Sánchez 1992), el Test de Habilidades para la Gestión en la Negociación de Conflictos (THGNC) (Vicuña, Hernández, Paredes y Ríos, 2008), y la Escala de Tipo de Espectador de Violencia Entre Pares/Bullying (TEVEP/B) (Quintana et al., 2009).

De la revisión de estos instrumentos y su validación por juicio de expertos se precisa que el CEA consta de dos áreas: 1) logros académicos y 2) relaciones interpersonales (31 ítems). Para efectos de la presente investigación sólo se utilizará la 2. ${ }^{\text {da }}$ Escala. Así mismo, en cuanto al THGNC, se encontró que la dimensión Perspectiva considerada en la prueba original no se ajusta con las habilidades que estamos investigando dentro del contexto de la negociación de conflictos, lo cual llevó a reemplazar la misma por la habilidad denominada "Cognitiva interpersonal" que mide la capacidad de conocer las causas, generar alternativas de solución y planificar su ejecución, considerando las consecuencias en los participantes en conflictos interpersonales, en el sentido del llamado "Pensamiento planificador medios-fines", argumentado por Bethencourt (1997), conceptualizado a partir de las hipótesis a 
este respecto de Spivack, Platt y Shure (1976), sistematizadas e interpretadas en el enfoque cognitivo-conductual por Pelenacho (1989).

En razón a estas consideraciones se pueden proponer las siguientes hipótesis:

1. Existe relación significativa entre los estilos explicativos y las habilidades de gestión para la negociación de conflictos en adolescentes espectadores de violencia entre pares (bullying).

2. Cada uno de los diferentes estilos explicativos se relaciona significativamente con cada uno de los tipos de espectador de violencia entre pares (bullying).

3. Cada una de las dimensiones de la gestión para la negociación de conflictos se relaciona significativamente con cada uno de los tipos de espectador de violencia entre pares (bullying).

4. Cada uno de los diferentes estilos explicativos se relaciona significativamente con cada una de las dimensiones de la gestión para la negociación de conflictos.

\section{MÉTODO}

\section{Muestra}

La muestra está conformada por 600 estudiantes adolescentes procedentes de centros educativos de Lima; representativos de los tres tipos identificados de espectadores de bullying.

El diseño para la observacion y control de las variables extrañas se podría caracterizar como de investigación básica (explicativo), descriptivo-comparativo y de tipo transversal. Teniendo como Variables Independientes a los Estilos explicativos y las Habilidades para la gestión de negociación de conflictos y como Variable Dependiente a los Tipos de Espectadores de bullying, en adolescentes de 14 a 18 años, de ambos sexos, estudiantes de secundaria de distintos niveles socioecónomicos residentes en Lima Metropolitana.

\section{Técnicas e instrumentos de recolección de datos}

Ficha demográfica: Permite registrar algunas características relevantes de los sujetos estudiados: Lugar de residencia, Edad, Sexo, Grado de instrucción, etc.

Cuestionario de Estilos Atributivos CEA (Sánchez, 1992): Consiste de dos áreas: 1) logros académicos (41 ítems) y 2) relaciones interpersonales (31 ítems). Para efectos de la presente investigación, sólo utilizaremos la segunda, relacionada con factores de éxito o fracaso (subescalas $8,9,10,11$ y 12). Sus respuestas se hacen en base a una gradación de puntajes de 0 a 4 .

Definición conceptual: Las distintas causas a las que pueden atribuirse los hechos observados en relación con la propia conducta pueden clasificarse dentro de distintas dimensiones: interno-externo, estable-variable, controlable-no controlable, general-específico (Weiner, 1979, 1986; Alonso Tapia, 1983); los estilos atributivos pueden definirse en función de tales dimensiones causales. 
Definición Operacional: Los estilos atributivos acerca de las relaciones interpersonales que mide este instrumento son:

Internalización del fracaso en las relaciones interpersonales. Se atribuye el fracaso a causas internas, a la falta de esfuerzo o de habilidad.

Atribución del éxito en las relaciones interpersonales al esfuerzo (causas internas).

Externalización del éxito en las relaciones interpersonales. El éxito se atribuye a causas externas como la suerte o los otros.

Atribución del éxito en las relaciones interpersonales a la habilidad. Refleja la tendencia a internalizar el éxito.

Externalización del fracaso. El contenido de los mismos hace referencia a la atribución del fracaso a la mala suerte o a acción de otras personas, esto es, a causas externas.

Con Coeficiente de Validez de Aiken de los ítems que oscilaron predominantemente entre 0.70 y 1.00 . La confiabiliadad obtiene por el alfa de Cronbach de $r=.718$ estadísticamente significativa al .001.

Test de Habilidades para la Gestión en la Negociación de Conflictos (Vicuña, Hernández, Paredes y Ríos, 2008) Adaptado por los subscritos. Cuenta con 50 ítems a responderse en gradación de puntajes de 1 a 6 , desde "definitivamente en desacuerdo" hasta "definitivamente de acuerdo". Las habilidades para la gestion de conflictos interpersonales que mide este instrumento, reformuladas por los argumentos vertidos líneas arriba son:

Definición conceptual: Habilidades para destacar lo valioso de los puntos de vista o situaciones en conflicto, haciéndoles entender que, a la luz de las circunstancias, de los recursos y del momento actual, sólo es posible asumir un punto de vista o situación y asegurándose que este proceso sea asimilado con tolerancia por las partes, siendo capaz de contrarrestar las reacciones emocionales que surgieran.

Definición operacional: Las habilidades para la gestión en la negociación de conflictos son:

Comunicación, Capacidad para usar eficazmente herramientas de comunicación verbal y no verbal, que se expresan en cómo se escucha, cómo se responde gestual, postural y verbalmente.

Compromiso, Capacidad para involucrarse con el conflicto, en el sentido de sentirse cómodo participando en su solución, identificándose con el papel de negociador.

Cognición interpersonal, Capacidad de conocer las causas, generar alternativas de solución y planificar su ejecución, considerando las consecuencias en los participantes en conflictos interpersonales.

Control emocional, Capacidad para usar sus recursos personales o potenciales para evitar que la carga emocional que le llega termine por bloquearlo emocionalmente, irritándolo o asumiendo posturas preferenciales, o estresándolo, y por ende disminuyendo su eficiencia y eficacia negociadora.

Empatía, Capacidad para abandonar sus puntos de vista y asumir en cada instante los puntos de vista de la persona o las personas en conflicto. 
Con Coeficiente de Validez de Aiken de los ítems que oscilaron predominantemente entre 0.80 y 1.00 . La confiabiliadad obtiene por el alfa de Cronbach de $\mathrm{r}=.830$ estadísticamente significativa al .001 .

Escala de Tipo de Espectador de Violencia Entre Pares (Quintana, Montgomery y Malaver, 2009), Es una escala de 30 ítems originales, calificados en función a su frecuencia: desde "siempre" (3) a "nunca" (0), que Definición Operacional: Permite identificar tres tipos de adolescentes espectadores de violencia entre pares: Prosocial, indiferenteculpabilizado y antisocial, correspondientes a los que Monjas y Aviles (2006) denominan Prosociales, espectadores propiamente dichos y antisociales, descritos líneas arriba.

Con Coeficiente de Validez de Aiken de los ítems que oscilaron predominantemente entre 0.80 y 1.00. La confiabiliadad obtiene por el alfa de Cronbach de $\mathrm{r}=.626$ estadísticamente significativa al .001 .

Para efectos de la Recolección de datos se procedió con las siguientes actividades: Se coordinó con las autoridades de los centros educativos previstos para ser integrados en el estudio, se fue en sucesivas ocasiones a los centros que accedieron a colaborar con el estudio y se administró en forma anónima el Cuestionario de Estilos Atributivos, el Test de Habilidades para la Gestión en la Negociación de Conflictos y la Escala de Tipo de Espectador de Violencia Entre Pares a adolescentes que cumplían los criterios de inclusión en la muestra. Finalmente se elaboró la base de datos, depurando a aquellos sujetos que a pesar de las indicaciones no hubieran completado el llenado total de todos los instrumentos considerados.

Posteriormente se procedió a Procesar y analizar los datos para contrastar las hipótesis propuestas con los estadísticos: Análisis de Varianza de varios factores y el Coeficiente de Correlación Tau Kendall. Y para efectos de estimar la validez y confiabilidad de los instrumentos usados el coeficiente alpha de Crombach y el Análisis Factorial, todas ellas con el Paquete estadístico SPSS N. ${ }^{0} 16$.

\section{RESULTADOS}

A continuación se presentarán los resultados en relación a cada una de las hipótesis formuladas, a saber:

En relación a si cada uno de los diferentes estilos explicativos se relaciona significativamente con cada uno de los tipos de espectador de violencia entre pares (bullying). 
TABLA 1. Estilos explicativos en relación al Espectador Prosocial.

\begin{tabular}{|c|c|c|c|c|c|c|}
\hline & & $\begin{array}{l}\text { Atribuye a } \\
\text { si mismo } \\
\text { el fracaso }\end{array}$ & $\begin{array}{c}\text { Atribuye a } \\
\text { su } \\
\text { esfuerzo el } \\
\text { éxito }\end{array}$ & $\begin{array}{l}\text { Atribuye a } \\
\text { causas } \\
\text { ajenas a si } \\
\text { mismo el } \\
\text { éxito }\end{array}$ & $\begin{array}{c}\text { Atribuye a } \\
\text { su } \\
\text { habilidad el } \\
\text { éxito }\end{array}$ & $\begin{array}{c}\text { Atribuye a } \\
\text { causas } \\
\text { ajenas a si } \\
\text { mismo el } \\
\text { fracaso }\end{array}$ \\
\hline & $\begin{array}{c}\text { Coeficiente } \\
\text { de } \\
\text { correlación }\end{array}$ & -.031 & $.169^{\star \star \star}$ & -.026 &, $172^{\star \star \star}$ & .059 \\
\hline \multirow{3}{*}{$\begin{array}{c}\text { Espectador Pro } \\
\text { social }\end{array}$} & Sig. Bilateral & .304 & .000 & .387 & .000 & .051 \\
\hline & $\mathrm{N}$ & 546 & 546 & 546 & 546 & 546 \\
\hline & $\begin{array}{c}\text { Coeficiente } \\
\text { de } \\
\text { correlación }\end{array}$ & $.127^{\star *}$ & .014 & $.073^{\star}$ & -.001 & $-.198^{\star *}$ \\
\hline \multirow{2}{*}{$\begin{array}{l}\text { Espectador } \\
\text { indiferente / } \\
\text { culpabilizado }\end{array}$} & Sig. Bilateral & .000 & .658 & .018 & .983 & .000 \\
\hline & $\mathrm{N}$ & 546 & 546 & 546 & 546 & 546 \\
\hline \multirow[t]{3}{*}{$\begin{array}{l}\text { Espectador } \\
\text { Amoral }\end{array}$} & $\begin{array}{c}\text { Coeficiente } \\
\text { de } \\
\text { correlación }\end{array}$ & $.062^{\star}$ & $-.060^{\star}$ & $.141^{\star \star \star}$ & -.051 & $.068^{\star}$ \\
\hline & Sig. Bilateral & .041 & .049 & .000 & .095 & .024 \\
\hline & N & 546 & 546 & 546 & 546 & 546 \\
\hline
\end{tabular}

En relación a si cada una de las dimensiones de la gestión para la negociación de conflictos interpersonales se relaciona significativamente con cada uno de los tipos de espectador de violencia entre pares (bullying).

TABLA 2. Dimensiones de la gestión para la negociación de conflictos interpersonales en relación a los tipos de Espectadores de Bullying

\begin{tabular}{|c|c|c|c|c|c|c|}
\hline & & Comunicadón & Compromiso & $\begin{array}{c}\text { Cognición } \\
\text { interpersonal }\end{array}$ & $\begin{array}{c}\text { Control } \\
\text { Emocional }\end{array}$ & Empatia \\
\hline \multirow{3}{*}{$\begin{array}{l}\text { Espectador } \\
\text { Prosocial }\end{array}$} & $\begin{array}{c}\text { Coeficiente } \\
\text { de } \\
\text { correlación }\end{array}$ & $192^{\star \star}$ & $109^{\star \star}$ & $231^{\star \star}$ & $167^{\star \star}$ & $106^{*}$ \\
\hline & Sig. Bilateral & .000 & .000 & .000 & .000 & .000 \\
\hline & $N$ & 546 & 546 & 546 & 546 & 546 \\
\hline \multirow{3}{*}{$\begin{array}{l}\text { Espectador } \\
\text { indiferente I } \\
\text { culpabilizado }\end{array}$} & $\begin{array}{c}\text { de } \\
\text { correlación }\end{array}$ & $.098^{\star \star}$ & .037 & .057 & .039 & $.067^{\star \star}$ \\
\hline & Sig. Bilateral & .001 & .225 & .061 & 199 & .028 \\
\hline & $\stackrel{\mathrm{N}}{\mathrm{N}}$ & 546 & 546 & 546 & 546 & 546 \\
\hline \multirow{3}{*}{$\begin{array}{l}\text { Espectador } \\
\text { Amoral }\end{array}$} & $\begin{array}{c}\text { de } \\
\text { correlación }\end{array}$ & -033 & 000 & -.073 & .003 & 015 \\
\hline & Sig. Bilateral & .269 & .991 & .016 & 919 & 610 \\
\hline & $\mathrm{N}$ & 546 & 546 & 546 & 546 & 546 \\
\hline
\end{tabular}

- Correlación Tau-bde Kendall significativa a nivel 0,01 (bilateral)

"Correlación Tau-b de Kendall significativa a nivel 0,05 (bilateral) 
En relación a si cada uno de los diferentes estilos explicativos se relaciona significativamente con cada una de las dimensiones de la gestión para la negociación de conflictos interpersonales.

TABLA 3. Estilos explicativos en relación a las dimensiones de la gestión para la negociación de conflictos interpersonales.

\begin{tabular}{lllllll}
\multicolumn{7}{c}{ Correlaciones } \\
\hline
\end{tabular}

"x. La comrelación es significativa al nivel 0,01

*. L correlación es significativa al nivel 0,05

Como se puede inferir de las Tablas 1,2 y 3 , los resultados en líneas generales evidencian la comprobación de la hipótesis general de que existe relación significativa entre los estilos explicativos y las habilidades de gestión para la negociación de conflictos en adolescentes espectadores de violencia entre pares (bullying).

Además, para ratificar estos hallazgos se usó el análisis de varianza encontrando diferencias estadísticamente significativas entre los tipos de espectadores prosocial e indiferente/ culpabilizado en 3 de los 5 estilos atributivos y en 3 de las 5 habilidades para la gestión de conflictos interpersonales, a saber, Atribuir el fracaso tanto a sí mismo, como a causas ajenas a sí mismo, con la predominancia del espectador indiferente/culpabilizado (Medias de la diferencia de -1.28743 , sig. al .038 y de -1.4972 , sig al .024, respectivamente) y así tambien en el estilo atribuye a su habilidad el éxito, con la predominancia del espectador prosocial (Media de la diferencia de .96408, sig. al .043), como puede observarse en la Tabla 4. 
TABLA 4. Anova de Estilos explicativos según el Tipo de Espectador de Violencia entre pares

\begin{tabular}{|c|c|c|c|c|c|c|c|}
\hline \multirow[b]{2}{*}{ DenendentVariable } & \multirow[b]{2}{*}{ (I) TinOEVEP } & \multirow[b]{2}{*}{ (D) TinoEVEP } & \multirow{2}{*}{$\begin{array}{c}\text { Mean } \\
\text { Difference (f- } \\
J)\end{array}$} & \multirow[b]{2}{*}{ Std. Error } & \multirow[b]{2}{*}{ Sig. } & \multicolumn{2}{|c|}{$95 \%$ Confidence Interval } \\
\hline & & & & & & Lower Bound & Upper Bound \\
\hline \multirow{6}{*}{$\begin{array}{l}\text { Atribuye a si mismo el } \\
\text { Fracaso }\end{array}$} & \multirow[t]{2}{*}{ Prosocial } & Indiferenteiculpabilizado & $-1,28743^{\prime}$ & .50123 & .038 & $-2,5181$ &,- 0568 \\
\hline & & Amoral & .66684 & .52903 & .452 & $-1,9657$ & .6320 \\
\hline & \multirow[t]{2}{*}{ Indiferente/culpabilizado } & Prosocial & $1,28743^{\prime}$ & .50123 & .038 & .0568 & 2,5181 \\
\hline & & Amoral & .62059 & .65981 & .643 & .9994 & 2,2405 \\
\hline & \multirow[t]{2}{*}{ Amoral } & Prosocial & .66684 & .52903 & .452 & -6320 & 1,9657 \\
\hline & & Indiferente/culpabilizado & -62059 & .65981 & .643 & $-2,2405$ & .9994 \\
\hline \multirow{6}{*}{$\begin{array}{l}\text { Atribuye a su esfuerzo el } \\
\text { Exito }\end{array}$} & \multirow[t]{2}{*}{ Prosocial } & Indiferente/culpabilizado & 1,07791 &, 45180 & .059 & -0314 & 2,1872 \\
\hline & & Amoral & 1.02019 & .47686 & .103 & -.1506 & 2.1910 \\
\hline & \multirow[t]{2}{*}{ Indiferente/culpabilizado } & Prosocial & $-1,07791$ & .45180 & .059 & $-2,1872$ &, 0314 \\
\hline & & Amoral &,- 05773 & .59474 & .995 & $-1,5179$ & 1,4025 \\
\hline & \multirow[t]{2}{*}{ Amoral } & Prosocial & $-1,02019$ & .47686 & .103 & $-2,1910$ & .1506 \\
\hline & & Indiferenteiculpabilizado & 05773 & .59474 & .995 & $-1,4025$ & 1,5179 \\
\hline \multirow{6}{*}{$\begin{array}{l}\text { Atribuye a causas ajenas } \\
\text { a si mismo el Exito }\end{array}$} & \multirow[t]{2}{*}{ Prosocial } & Indiferenteiculpabilizado &,- 37525 & .44042 & .696 & $-1,4566$ & .7061 \\
\hline & & Amoral & .98854 & .46484 & .105 & $-2,1298$ &, 1527 \\
\hline & \multirow[t]{2}{*}{ Indiferente/culpabilizado } & Prosocial & .37525 & .44042 & .696 & 7061 & 1,4566 \\
\hline & & Amoral &., 61328 & .57975 & .572 & $-2,0367$ & .8101 \\
\hline & \multirow[t]{2}{*}{ Amoral } & Prosocial &, 98854 & .46484 & .105 & -1527 & 2,1298 \\
\hline & & Indiferente/culpabilizado & .61328 & .57975 & .572 & -8101 & 2,0367 \\
\hline \multirow{6}{*}{$\begin{array}{l}\text { Atribuye a su habilidad el } \\
\text { Exito }\end{array}$} & \multirow[t]{2}{*}{ Prosocial } & Indiferenteiculpabilizado & $96408^{\circ}$ & .38309 & .043 & .0235 & 1,9046 \\
\hline & & Amoral & .53198 & .40434 & .421 & -4608 & 1,5247 \\
\hline & \multirow[t]{2}{*}{ Indiferente/culpabilizado } & Prosocial & $-96408^{\circ}$ & .38309 & .043 & $-1,9046$ & -0235 \\
\hline & & Amoral & -.43210 & .50429 & .693 & -1.6702 & .8060 \\
\hline & \multirow[t]{2}{*}{ Amoral } & Prosocial &,- 53198 & .40434 & .421 & $-1,5247$ & .4608 \\
\hline & & Indiferente/culpabilizado & .43210 & .50429 & .693 & -8060 & 1,6702 \\
\hline \multirow{6}{*}{$\begin{array}{l}\text { Atribuye a causas ajenas } \\
\text { a si mismo el Fracaso }\end{array}$} & \multirow[t]{2}{*}{ Prosocial } & Indiferente/culpabilizado & $-1,49472^{\circ}$ & .54471 & .024 & $-2,8321$ & -1573 \\
\hline & & Amoral & -.22138 & .57493 & .929 & $-1,6329$ & 1,1902 \\
\hline & \multirow[t]{2}{*}{ Indiferente/culpabilizado } & Prosocial & $1,49472^{\circ}$ & .54471 & .024 & .1573 & 2,8321 \\
\hline & & Amoral & 1.27334 & .71705 & .208 & -4871 & 3,0338 \\
\hline & \multirow[t]{2}{*}{ Amoral } & Prosocial & .22138 & .57493 & .929 & $-1,1902$ & 1,6329 \\
\hline & & Indiferente/culpabilizado & $-1,27334$ & 71705 & 208 & $-3,0338$ & 4871 \\
\hline
\end{tabular}

*. The mean difference is significant at the 0.05 level.

Así mismo, también mediante el análisis de varianza se corroboran diferencias estadísticamente significativas entre los tipos de espectadores prosocial y el amoral en 3 de las 5 habilidades para la gestión de conflictos interpersonales, a saber, en cuanto a comunicación, cognición interpersonal y control emocional (Medias de la diferencia de 1.78484, sig al .035; de 2.40010, sig al .002 y de 1.65485, sig al .035, respectivamente), como puede observarse en la Tabla 5. 
TABLA 5. Anova de las dimensiones de la gestión para la negociación de conflictos interpersonales según el Tipo de Espectador de Violencia entre pares

\begin{tabular}{|c|c|c|c|c|c|c|c|}
\hline \multirow[b]{2}{*}{ DependentVariable } & \multirow[b]{2}{*}{ (1) TinoEVEP } & \multirow[b]{2}{*}{ (J) TIROEYEP } & \multirow[b]{2}{*}{$\begin{array}{c}\text { Mean } \\
\text { Difference (1- } \\
J)\end{array}$} & \multirow[b]{2}{*}{ Std. Error } & \multirow[b]{2}{*}{ Sia. } & \multicolumn{2}{|c|}{$95 \%$ Confidence Interval } \\
\hline & & & & & & Lower Bound & Unper Bound \\
\hline \multirow[t]{6}{*}{ Comunicación } & Prosocial & Indiferente/culpabilizado & .61409 & .65157 & .642 &., 9856 & 2,2138 \\
\hline & & Amoral & $1,78484^{\circ}$ & .68770 & .035 & .0964 & 3.4733 \\
\hline & Indiferentelculpabilizado & Prosocial &,- 61409 & .65157 & .642 & $-2,2138$ & .9856 \\
\hline & & Amoral & 1.17075 & .85770 & .395 & .9351 & 3.2766 \\
\hline & Amoral & Prosocial & $-1,78484^{\circ}$ & .68770 & .035 & $-3,4733$ & -0964 \\
\hline & & Indiferente/culpabilizado & -1.17075 & .85770 & .395 & -3.2766 & .9351 \\
\hline \multirow[t]{6}{*}{ Compromiso } & Prosocial & Indiferente/culpabilizado &, 85637 & .61344 & .378 &,- 6497 & 2,3625 \\
\hline & & Amoral & 1.17041 & .64746 & .196 & -.4192 & 2.7600 \\
\hline & Indiferente/culpabilizado & Prosocial &,- 85637 & .61344 & .378 & $-2,3625$ & .6497 \\
\hline & & Amoral & .31403 & .80751 & .927 & -1.6686 & 2.2966 \\
\hline & Amoral & Prosocial & $-1,17041$ & .64746 & .196 & $-2,7600$ & .4192 \\
\hline & & Indiferente/culpabilizado & -.31403 & .80751 & .927 & -2.2966 & 1.6686 \\
\hline \multirow[t]{6}{*}{ Cognicion Interpersonal } & Prosocial & Indiferente/culpabilizado & 1,36793 & .62797 & .094 & -1739 & 2,9097 \\
\hline & & Amoral & $2,40010^{\circ}$ & .66280 & .002 & .7728 & 4,0274 \\
\hline & Indiferente/culpabilizado & Prosocial & $-1,36793$ & .62797 & .094 & $-2,9097$ & .1739 \\
\hline & & Amoral & 1.03217 & .82664 & .459 & .9974 & 3.0617 \\
\hline & Amoral & Prosocial & $-2,40010^{\circ}$ & .66280 & .002 & $-4,0274$ & .7728 \\
\hline & & Indiferente/culpabilizado & $-1,03217$ & .82664 & .459 & $-3,0617$ & .9974 \\
\hline \multirow[t]{6}{*}{ Control Emocional } & Prosocial & Indiferente/culpabilizado & .78804 & .60236 & .426 & -6909 & 2,2670 \\
\hline & & Amoral & $1.65485^{\circ}$ & .63577 & .035 & .0939 & 3.2158 \\
\hline & Indiferentelculpabilizado & Prosocial &,- 78804 & .60236 & .426 & $-2,2670$ & .6909 \\
\hline & & Amoral & 86681 & .79293 & .551 & $-1,0800$ & 2,8136 \\
\hline & Amoral & Prosocial & $-1,65485^{\circ}$ & .63577 & .035 & $-3,2158$ & .0939 \\
\hline & & Indiferente/culpabilizado & -.86681 & .79293 & .551 & $-2,8136$ & 1.0800 \\
\hline \multirow[t]{6}{*}{ Empatia } & Prosocial & Indiferente/culpabilizado & .54012 & .58752 & .656 & -9023 & 1,9826 \\
\hline & & Amoral & .79225 & .62010 & .443 & .7302 & 2.3147 \\
\hline & Indiferente/culpabilizado & Prosocial &,- 54012 & .58752 & .656 & $-1,9826$ & .9023 \\
\hline & & Amoral & .25213 & .77339 & .948 & $-1,6467$ & 2,1510 \\
\hline & Amoral & Prosocial &,- 79225 & .62010 & .443 & $-2,3147$ & .7302 \\
\hline & & Indiferente/culpabilizado & -25213 & .77339 & 948 & $-2,1510$ & 1,6467 \\
\hline
\end{tabular}

\section{DISCUSIÓN}

\section{Respecto a los estilos atributivos de los tipos de espectadores de bullying}

Al analizar, en función a los resultados estadísticos consignados líneas arriba, acerca de cómo procesan los espectadores de bullying las causas de su comportamiento, se visualiza que utilizan diferentes Estilos Atributivos en función de cómo perciben su conducta al establecer relaciones interpersonales.

Espectador Prosocial: muestra una correlación significativa positiva (Tabla 1):

1. Cuando el éxito es considerado como respuesta a su esfuerzo y perseverancia para establecer y mantener relaciones interpersonales, mostrando mayor proactividad para desarrollarlas (.169, sig al 0.00).

2. Así también con el estilo atributivo en el cual se percibe como generador del éxito en sus relaciones producto de su habilidad, competencia social. Esta percepción lo estimula a desarrollar una actitud positiva para vincularse tanto con su grupo de pares, como con su entorno (.172, sig al 0.01). 
Espectador Indiferente - Culpabilizado: muestra una correlación significativa positiva (Tabla 1):

1. Con el estilo atributivo cuya dimensión causal determina el fracaso en el establecimiento de relaciones interpersonales por factores internos, tendiendo a mostrar una actitud pasiva - evitativa para vincularse, afín de eludir el afrontar conflictos con su grupo de pares (.127, sig al 0.01).

2. En atribuir el éxito en sus relaciones interpersonales, a factores externos, debido a causas situacionales, como el ser aceptado por los demás, a la disposición que tengan los otros para aproximarse a él, ó también producto del azar, la suerte (.073, sig al 0.05).

3. A externalizar el fracaso en el desarrollo de sus relaciones interpersonales, tendiendo a adjudicar a los demás su deficiente competencia social (.198, sig al 0.01).

Espectador Amoral: muestra una correlación significativa positiva (Tabla 1):

1. Para atribuir el fracaso en sus relaciones interpersonales a factores internos, como su inhabilidad para comunicarse, ó para mantener el esfuerzo en entablar una relación amical con su grupo de pares (.062, sig al 0.05).

2. Con el estilo atributivo cuya dimensión causal determina el éxito en las relaciones interpersonales por factores externos, el espectador amoral percibe al azar, ó a la aceptación del entorno (.141, sig al 0.01), Ej. su grupo de pares, como facilitadores en el establecimiento de relaciones amicales.

3. En atribuir a causas ajenas a sí mismo el fracaso (.068, sig al 0.05), considerando al entorno como causal de sus fracasos interpersonales, lo cual se condice por la actitud complaciente que muestra ante el maltrato entre pares, que podría dar lugar a una actitud rechazo de los demás.

Espectador Amoral: muestra una correlación significativa negativa (Tabla 1):

1. Con atribuir al esfuerzo de sí mismo el éxito (-.060, sig al 0.05), lo que se condice con su tendencia a considerar al entorno como causal de sus éxitos interpersonales.

\section{Respecto a las habilidades para la negociación de conflictos de los tipos de espec- tadores de bullying}

Al analizar Habilidades para la Gestión en la Negociación de Conflictos de los Adolescentes en los distintos tipos de espectadores encontramos

Espectador Prosocial: muestra una correlación significativa positiva (Tabla 2):

1. Con la habilidad de comunicación, presentando un mayor desarrollo de sus habilidades compresivas, expresivas, de escucha activa.

2. Con la habilidad de compromiso, es decir, se involucra, muestra iniciativa, proactividad, para afrontar una situación de conflicto interpersonal.

3. Con relación a la habilidad cognitiva interpersonal, lo cual se corrobora con el uso de estrategias de afrontamiento activo, de planificación para la generación de alternativas 
de solución y su ejecución considerando las consecuencias en los participantes en los conflictos interpersonales.

4. Con la habilidad de empatía, es decir, es más receptivo, colocándose y comprendiendo el punto de vista de los actores participantes en una situación de conflicto.

5. Con la habilidad de control el espectador prosocial, hace uso de estrategias que le permiten regular las reacciones emocionales que emergen de situaciones contradictorias o conflictivas.

Espectador Indiferente/culpabilizado: muestra una correlación significativa positiva (Tabla 2):

1. Con la habilidad de comunicación, presentando un mayor desarrollo de sus habilidades compresivas, expresivas, de escucha activa.

2. Con la habilidad de empatía, es decir, es más receptivo, colocándose y comprendiendo el punto de vista de los actores participantes en una situación de conflicto.

3. Pero al no contar con la habilidad de planificación para la generación de alternativas de solución y su ejecución considerando las consecuencias en los participantes en los conflictos interpersonales, la habilidad de comprometerse e involucrarse para afrontar una situación de conflicto interpersonal, va a tender a mantenerse al margen de la problemática del bullying.

Espectador Amoral: muestra una correlación significativa negativa (Tabla 2):

1. Con respecto a la habilidad cognitiva interpersonal, es decir, mientras más actitudes de aceptación del maltrato entre pares evidencian estos jóvenes, menos probable es que presenten estrategias adecuadas de planificación, ejecución, regulación para afrontar y resolver situaciones que impliquen conflictos interpersonales.

Los resultados de esta investigación como de las otras que venimos realizando en esta línea de trabajo nos reafirman que se hace necesario enfocar al bullying como un fenómeno sistémico en donde no sólo debe circunscribirse la dinámica de este tipo de violencia en la escuela a la triada víctima-victimario-espectador dentro del círculo de grupo de pares, sino que involucra en su abordaje o invisibilización, en su reforzamiento o afrontamiento los otros miembros de la comunidad educativa. Por lo cual es fundamental conocer las percepciones, modos como gestionan los conflictos los docentes y directivos de las instituciones, así como el clima institucional que se genera. Ya que la comunidad educativa puede tornarse en un factor de riesgo induciendo, manteniendo, incrementando un Clima de Violencia o constituyéndose en un factor protector para promover una Cultura de Paz.

\section{CONCLUSIONES}

Tal como lo postulaban las hipótesis, se ha encontrado que:

2. Existe una correlación directa y significativa entre el espectador prosocial y todas las habilidades para la gestión de conflictos interpersonales, mientras que, por el contrario,

3. Los espectadores amoral e indiferente-culpabilizado no guardan correlación con las habilidades para la gestión de conflictos interpersonales, a excepción de la habilidad 
cognitiva interpersonal con la cual el espectador Amoral muestra una correlación inversa y significativa.

4. El espectador prosocial correlaciona positiva y significativamente, con atribuir a su propio esfuerzo y habilidad sus éxitos en las relaciones interpersonales.

5. Los espectadores amoral e indiferente-culpabilizado, por el contrario, correlacionan significativamente con atribuir a causas ajenas a sí mismos sus éxitos y fracasos, así como a atribuirse a sí mismos sus fracasos.

\section{REFERENCIAS BIBLIOGRÁFICAS}

1. Avilés, J. (2006). Bullying: El maltrato entre iguales. Salamanca: Amaru.

2. Bethencourt, J. (1997). El Pensamiento Planificador Medios-Fines: Una investigación sobre la validez de un test para medirlo. Revista Latinoamericana de Psicología, 29 (2), 321-339

3. Linares, B (2007). Ansiedad y agresión en jóvenes de 12 a 16 años: Comorbilidad, estilos atribucionales y funcionamiento adaptativo social. Tesis de Maestría para la Fundación Universitaria Konrad Lorenz, Bogotá.

4. Malaret, J. (2001). Manual de negociación y mediación. Madrid: Colex.

5. Matalinares, M. Tueros, R. y Yaringaño, J. (2009). Adaptación psicométrica del Cuestionario de Estilos Atributivos. Revista IIPSI, 12(1), 173-189.

6. Pelechano, V. (1989) Inteligencia y habilidades interpersonales; un diseño de modelo de trabajo y algunas hipótesis contrastables. En Inteligencia y Cognición: Homenaje al profesor Mariano Yela Ganizo. Madrid: Editorial Complutense.

7. Quintana, A., Montgomery, W. y Malaver, C. (2009). Modos de afrontamiento y conducta resiliente en adolescentes espectadores de violencia entre pares. Revista IIPSI, 12(1), 153-171.

8. Quintana, A., Montgomery, W., Malaver, C. y Gabriela. R. (2010). Capacidad de disfrute y percepción del apoyo comunitario en espectadores de episodios de violencia entre pares. Investigación. Revista IIPSI, 13(1), 139-150.

9. San Juan, P. y Magallares, A. (2007). Estilos explicativos y estrategias de afrontamiento. Clínica y Salud, 18(1), 83-98.

10. Staats, A.W. (1997). Conducta y personalidad: Conductismo psicológico. Bilbao: Descleé de Brouwer.

11. Vicuña, L. Hernández, H., Paredes, M. y Ríos, J. (2008). Elaboración del Test de Habilidades para la Gestión en Negociación de Conflictos. Revista IIPSI, 11(2), 183-200.

12. Visdómine-Lozano, J.C. y Luciano, C. (2006). Locus de control y autorregulación conductual: Revisiones conceptual y experimental. International Journal of Clinical and Health Psychology, 6(3), 729-751. 